$14 \mid 1992$

Varia

\title{
Documents relatifs aux lettres de A. Dettonville (I)
}

Pascal et le Père Tacquet

Dominique Descotes

\section{OpenEdition}

Journals

Édition électronique

URL : http://journals.openedition.org/ccibp/619

DOI : $10.4000 /$ ccibp. 619

ISSN : 2493-7460

Éditeur

Centre international Blaise Pascal

\section{Édition imprimée}

Date de publication : 5 février 1992

Pagination : 18-23

ISSN : 0249-6674

Référence électronique

Dominique Descotes, «Documents relatifs aux lettres de A. Dettonville (I) », Courrier du Centre

international Blaise Pascal [En ligne], 14 | 1992, mis en ligne le 07 janvier 2016, consulté le 02 mai 2019.

URL : http://journals.openedition.org/ccibp/619; DOI : 10.4000/ccibp.619

Ce document a été généré automatiquement le 2 mai 2019.

Centre international Blaise Pascal 


\section{Documents relatifs aux lettres de A. Dettonville (I)}

Pascal et le Père Tacquet

Dominique Descotes

\section{Pascal, disciple du Père Tacquet?}

1 Le problème de la dette de Pascal envers le Jésuite André Tacquet a été soulevé par un autre Jésuite, le Père Bosmans.

2 Sa thèse est sommairement la suivante. À l'époque où il compose la célèbre conclusion du Potestatum numericarum Summa sur les grandeurs de différents genres, Pascal n'a sur les indivisibles que des idées confuses. Lorsqu'il parle d'indivisibles géométriques, il les entend au sens strict de Cavalieri, comme éléments rigoureusement nuls : c'est ainsi qu'il écrit que «dans le cas d'une grandeur continue, des grandeurs d'un genre quelconque, ajoutées, en tel nombre qu'on voudra, à une grandeur d'un genre supérieur, ne l'augmentent de rien. Ainsi les points n'ajoutent rien aux lignes, les lignes rien aux surfaces, les surfaces aux solides ${ }^{1}$ ». Mais aussitôt Pascal ajoute que " pour employer le langage des nombres (...), les racines ne comptent pas par rapport aux carrés, les carrés par rapport aux cubes, les cubes par rapport aux carrés-carrés »; or dans ce cas, proteste le P. Bosmans, ce sont «des indivisibles qui ne sont plus nuls du tout (...). Ce sont simplement des quantités négligeables devant d'autres ${ }^{2} »$. Ces deux conceptions apparemment incompatibles voisinant dans le Potestatum numericarum Summa, le P. Bosmans conclut qu'un « écrivain aussi sûr de sa plume que Pascal, disant aussi aisément, aussi clairement (...) ce qu'il pense, quand il a une idée claire» n'aurait pas tenu un langage aussi confus «s'il avait déjà eu la notion exacte de la nature des indivisibles »; il faut donc supposer que Pascal n'avait lu à cette époque que les ouvrages de Cavalieri, dont le P. Bosmans pense reconnaître la marque dans la confusion de style et de pensée du Potestatum numericarum Summa. Le Jésuite souligne alors fortement la différence avec la netteté des Lettres de A. Dettonville où Pascal s'exprime avec une rigueur impeccable, substituant aux indivisibles stricts de Cavalieri des grandeurs homogènes dont les 
sommes diffèrent de la grandeur à mesurer d'une quantité moindre que toute donnée. Il trouve alors la raison de ce progrès dans la lecture que Pascal aurait faite entre 1654 et 1658 des livres publiés en 1651, pleins de "savante géométrie ", sur les cylindres et les anneaux, où le P. Tacquet "manie les indivisibles avec toute la rigueur désirable", lecture attestée par une référence dans un passage de la Lettre à Carcavy ${ }^{3}$. On trouve en effet dans les Cylindricorum et Annularium Libri IV deux Scholies, signalés d'ailleurs dès le XVII siècle par le Père Lalouvère, où le $P$. Tacquet critique la méthode des indivisibles qui procède par les "hétérogènes", en montrant par exemple qu'en considérant sans précaution les surfaces comme des sommes de lignes, on tombe dans des paralogismes ; si bien qu'une telle méthode n'est vraiment légitime que si elle est ramenée à celle des homogènes, c'est-à-dire aux inscriptions et circonscriptions des Anciens; cela n'a rien d'impossible dans la plupart des cas, comme l'attestent les démonstrations des deux genres que propose le P. Tacquet dans son traité. Comme Pascal développe des idées semblables dans les Lettres de A. Dettonville, le P. Bosmans conclut sans autre justification qu'il "n'a pas perdu la leçon» du Jésuite et lui doit sa nouvelle conception des indivisibles ${ }^{4}$.

3 À y regarder de près, cette argumentation ressemble un peu à une tentative de promotion du P. Tacquet par son confrère, aux dépens de Pascal. Car pour trouver matière à réflexion sur les rapports entre les indivisibles et la méthode des Anciens, Pascal n'avait pas nécessairement besoin de lire les Cylindricorum et Annularium Libri : dès ses premières recherches sur le vide, donc bien avant la rédaction du Potestatum numericarum Summa, il connaissait les œuvres de Torricelli ; il pouvait aussi s'en entretenir avec Roberval. Que les Scholies du P. Tacquet sur les dangers de la méthode de Cavalieri instruisent l'historien des mathématiques n'implique pas qu'ils aient apporté à Pascal une révélation : il a fort bien pu n'y trouver qu'une confirmation a posteriori de ses propres vues.

En fait, la thèse du Père Bosmans dépend étroitement de la chronologie des œuvres de Pascal proposée par l'édition dont il se sert, celle des Grands Écrivains de la France, surtout pour la date assignée à L'Esprit géométrique ${ }^{5}$. Cet opuscule exprime des positions très nettes et précises sur les indivisibles, les divisions indéfinies, les notions d'homogénéité et d'hétérogénéité, et le rapport entre grandeurs continues et discontinues, positions parfaitement compatibles avec les Lettres de A. Dettonville. Si, comme Brunschvicg, on date L'Esprit géométrique de 1657 ou 1658, immédiatement avant le concours de la Roulette, il est à la rigueur possible de supposer qu'il exprime aussi une conversion aux idées du P. Tacquet. Mais Jean Mesnard a établi que cet opuscule est beaucoup plus ancien, qu'il remonte à 1655 , ce qui le place au lendemain de la rédaction du Triangle arithmétique. Il en résulte que les idées de Pascal étaient fixées dès cette époque, et qu'il n'avait alors besoin ni du P. Tacquet ni d'un autre pour les rectifier' 


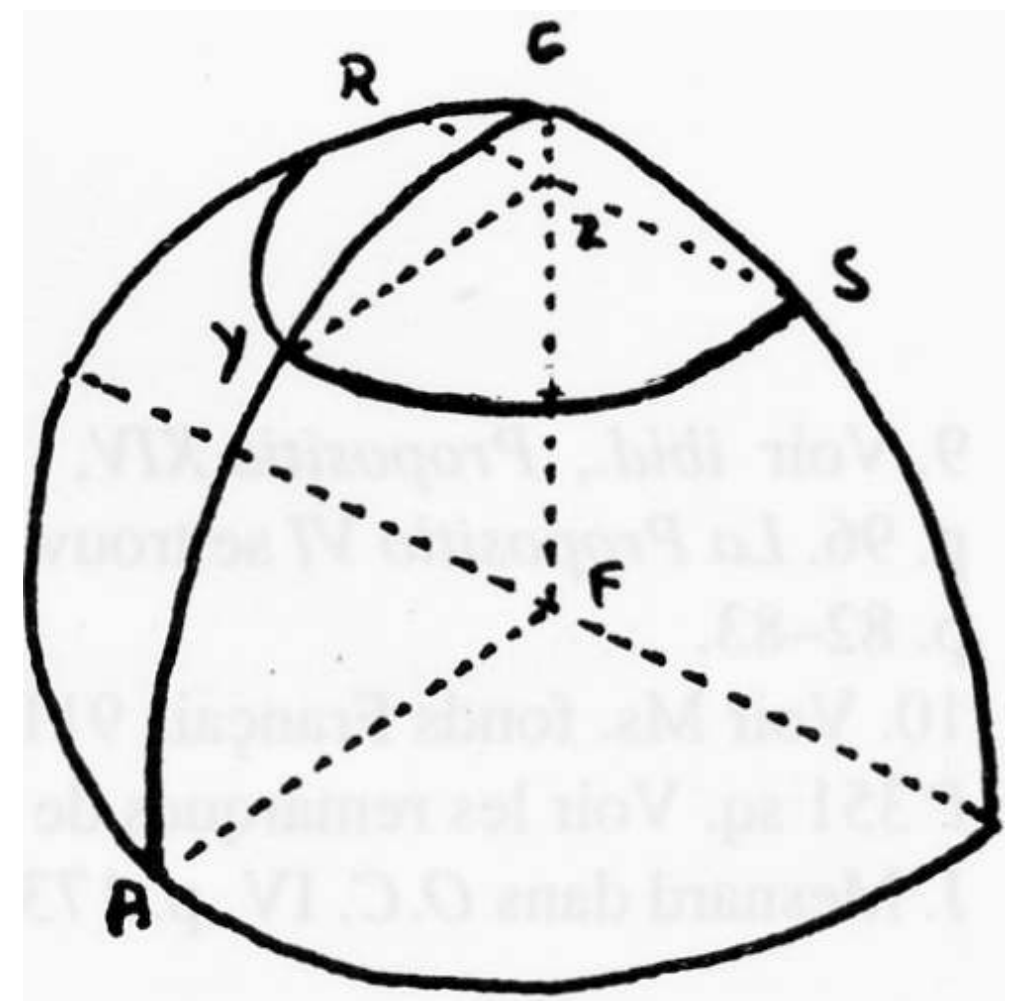

Euvres complètes, éd. Jean Mesnard, III, p.368 sq.

Est-il d'ailleurs si évident qu'il existe, entre le Potestatum numericarum Summa et les Lettres de A. Dettonville, une réelle différence de fond ? Les deux ouvrages s'adressent surtout à des lecteurs différents: la Lettre à Carcavy, avec son cortège d'Avertissements, vise les amateurs éclairés, à l'intention desquels Pascal multiplie les explications méthodologiques sur la règle d'homogénéité et les précautions à prendre pour ne pas comparer les incomparables; en revanche, le Postestatum numeriearum Summa en latin vise ceux "qui in indivisibilium doctrina tantisper versati sunt", auxquels il n'est pas besoin d'expliquer les fondements de la méthode ${ }^{7}$; là où le Père Bosmans voit une incohérence, il n'y a en fait qu'une expression abrégée, comme il convient dans une conclusion.

\section{Une référence de Dettonville}

Ces questions de méthode ont cependant détourné l'attention du point par lequel il aurait fallu commencer: la recherche du passage précis des Cylindricorum et Annularium Libri auquel renvoie la Lettre à Carcavy. Dettonville cite le nom du Père Tacquet, sans autre précision, dans le dernier mouvement de la Lettre, où il traite des rapports qui lient le double onglet d'un tri1igne rectangle et le demi-solide engendré par la rotation du même triligne autour de son axe. Il remarque que si le triligne CFA, dont la ligne courbe CA est monotone, tournant autour de CF, produit un solide, et si l'on coupe ce solide par des plans tous parallèles à sa base, on crée sur sa surface courbe des sections circulaires RYS. Dans la méthode des indivisibles, on envisage les tangentes aux arcs YY de la courbe CA : dans la rotation du triligne CFA autour de l'axe CF, ces tangentes engendrent de petites bandes circulaires elles-mêmes tangentes à la surface courbe du solide. Lorsque l'on 
multiplie indéfiniment le nombre des points de division $\mathrm{Y}$ de l'arc $\mathrm{CA}$, la somme des tangentes devient égale à la courbe CA elle-même. Et ainsi, lorsque le triligne tourne autour de son axe, la somme des bandes circulaires engendrées par les tangentes au quart de circonférence finit par s'identifier à la surface courbe du solide.

Schéma de la surface du demi-solide

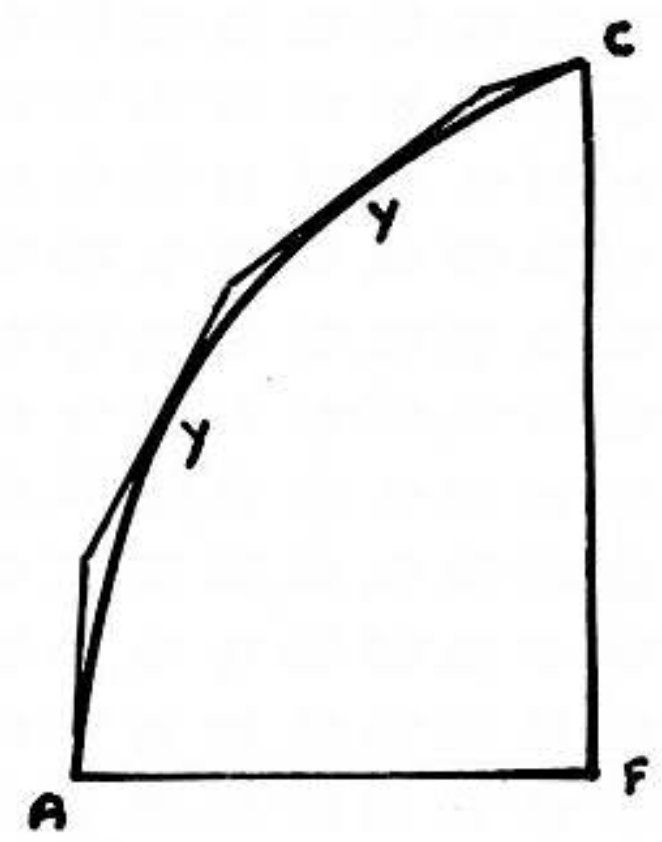

Euvres complètes, éd. Jean Mesnard, IV, p. 438

7 C'est là proprement ce que, d'après Dettonville, le $\mathrm{P}$. Tacquet a démontré : « La somme de ces demi-circonférences de la surface du demi-solide compose cette surface même (comme d'autres l'ont démontré, et entre autres le P. Tacquet ${ }^{8}$ ). »

On trouve en effet dans les Cylindricorum et Annularium Libri, Livre II, $1^{\text {re }}$ partie, une Proposition VI qui répond au propos de Pascal. Elle a pour objet de prouver que, si l'on considère dans le grand cercle BICQ d'une sphère, le demi-cercle BIC, auquel on inscrit et circonscrit des polygones réguliers, et si l'on fait tourner ces polygones autour du diamètre $\mathrm{BC}$, ils inscrivent et circonscrivent à la sphère des solides dont les surfaces diffèrent de celle de la sphère d'une quantité que l'on peut rendre aussi petite qu'on veut. Nous en donnons ci dessous la traduction. On voit en quoi elle s'accorde à la pensée de Pascal: les segments inscrits et circonscrits engendrent au cours de la rotation des bandes qui, à la limite, peuvent être dites composer la surface courbe. Conformément à ses principes, le $\mathrm{P}$. Tacquet le démontre à la manière des Anciens.

Cette référence pose cependant plusieurs problèmes. Le $\mathrm{P}$. Tacquet suppose en effet que BICQ est un cercle, donc le demi-solide une demi-sphère. Dans sa démonstration, il invoque d'ailleurs un théorème dû à Pappus, relatif à certaines propriétés des lignes inscrites et circonscrites au cercle. La proposition n'est donc concluante que dans ce cas particulier : «iis inscriptionibus ac circonscriptionibus sphaeram exhaurire ${ }^{9} »$. Il est vrai que, soucieux de généralité, le $\mathrm{P}$. Tacquet envisage ensuite les solides engendrés par la rotation d'une demi-ellipse, d'une parabole et d'une hyperbole, mais on chercherait en vain dans son ouvrage la démonstration que, dans ces cas-là, les inscriptions épuisent 
effectivement la surface courbe. Il en résulte que la référence fournie dans la Lettre à Carcavy est a fortiori insuffisante, puisque dans ce traité liminaire, Pascal suppose que la ligne courbe du triligne est quelconque (à condition d'être monotone). Pascal cite sans doute de mémoire, sans aller vérifier si, sur ce point tout à fait secondaire de sa démonstration, la proposition du P. Tacquet est vraiment suffisante.

L'expression "comme d'autres l'ont démontré, et entre autres le P. Tacquet » permet d'ailleurs de préciser cette conclusion. Quel autre? Le nom de Roberval se présente immédiatement : le manuscrit 9119 de la Bibliothèque Nationale contient une proposition sur la mesure de la surface sphérique où le géomètre établit qu'elle est composée par "l'agrégé des circonférences" formées sur les sinus de la courbe (autrement dit les rayons des cercles empilés qui forment la sphère). Pascal cite le P. Tacquet parce qu'il a incontestablement la priorité de publication; peut-être n'était-il pas mécontent de pouvoir en user ainsi, car la manie du secret de Roberval, son habitude de ne révéler ses découvertes qu'au compte-gouttes et de revendiquer après coup toutes les propositions produites par d'autres ont pu l'agacer quelque peu. On ne sait pas trop si Pascal a beaucoup apprécié d'entendre Roberval annoncer qu'avant même que Wren révèle sa rectification de la cycloïde, il la possédait dans ses papiers, alors que Pascal, lui, la recherchait vainement. En tout état de cause, Pascal pouvait à bon droit penser que ses écrits sur la roulette rendaient déjà à Roberval son dû et même peut-être plus, et que la référence au seul $P$. Tacquet était dès lors juste et suffisante ${ }^{10}$.

11 Il n'en demeure pas moins que, dans ces lignes de la Lettre à Carcavy, Pascal n'emploie pas à l'égard du Jésuite le style qui convient à l'égard d'un maître. De fait ce sont les dissemblances entre ces deux géomètres qui frappent, plus que leurs parentés. Le vocabulaire géométrique n'est pas le même : le Jésuite désigne par " portion cylindrique » ce que Dettonville appelle «onglet». Quant au fond, la méthode du P. Tacquet est purement géométrique, alors que Pascal se sert des nombres dans les problèmes de quadratures et de cubatures, ce qui lui permet de traiter des centres de gravité, auxquels le Jésuite ne touche pas. Celui-ci n'ignore pourtant pas la fécondité des suites numériques en géométrie, sa quadrature de la cycloïde en témoigne ; mais il ne s'en sert pas dans son grand traité. Si bien qu'au bout du compte on ne trouve aucune trace assurée d'une influence réelle $\mathrm{du}$ P. Tacquet sur Dettonville; il faut se contenter de constater une convergence de vue sur les indivisibles en général ${ }^{11}$.

\section{La dissertation sur la cycloïde}

12 À la suite de la Propositio VI, nous publions la traduction de la Dissertatio physico mathematica de Circulorum Volutionibus, que Pascal a pu lire à la suite de l'édition de 1651 des Cylindricorum et Annularium Libri. Elle contient une intéressante méthode de quadrature de la cycloïde. Pascal ne la mentionne pas dans l'Histoire de la Roulette; il n'avait pas à le faire, puisque le Père Tacquet ne revendique aucune priorité dans cette découverte, qu'il rend à ses sources, Torricelli et Descartes. La correspondance de Huygens révèle qu'en 1658 le Père Tacquet a su que la cycloïde faisait l'objet d'un concours ; mais sa santé l'empêcha d'approfondir les problèmes proposés par l'Anonyme ${ }^{12}$ . Nous ignorons donc ce que Pascal a pu penser de cette Dissertatio, mais il l'a presque certainement lue.

13 Sans entrer dans le commentaire théorique et critique de l'ouvrage, il faut indiquer brièvement l'intérêt qu'il peut présenter pour les pascalisants aujourd'hui. 

réflexions du Père Tacquet ont porté sur le paradoxe de la roue d'Aristote, discuté par des contemporains aussi prestigieux que Galilée, Descartes, Mersenne et Roberval. Chargé de l'instruction du comte Philippe Eugène de Hornes, Houterkercke et de Herlies, il lui fait soutenir publiquement des thèses sur ce sujet en 1650 au collège des Jésuites de Louvain. C'est à cet ancien élève que quelques années plus tard, il dédie ses Elementa Geometriae, pour lui rappeler cette soutenance ${ }^{13}$. La même année, il publie les résultats de ses réflexions dans une première version de la Dissertatio, qui fournit le texte des neuf thèses défendues par le comte, avec les démonstrations géométriques correspondantes. En 1651, le Jésuite publie une nouvelle rédaction à la suite de ses quatre livres sur les cylindres et les anneaux. C'est cette version, assez différente de la précédente, que Pascal a pu lire, et que nous traduisons. L'auteur semble avoir eu pour souci principal d'harmoniser la forme littéraire de sa Dissertatio avec celle de son grand traité. Il renonce à la forme scolaire des thèses pour donner toute son ampleur au style de démonstration géométrique par théorèmes, corollaires et scholies. Pour le fond, si dans l'ensemble le plan demeure à peu près le même qu'en 1650, il remanie considérablement le texte initial. En premier lieu, il l'allège de quelques développements jugés peut-être trop élémentaires ou insuffisamment originaux : il supprime par exemple les deux premières thèses ( Una ex omnibus corporibus sphaera in omnem loci differentiam moveri potest, nullâ loci factâ mutatione "; « in circula supra centrum circu acto, motus quôvis dato tardior; atque adeo omnia celeritatis possibilia decrementa exhibentur.»); disparaîtront aussi deux argumentations contre la méthodes des indivisibles, sans doute parce que l'auteur estime que ses livres sur les cylindres sont suffisants là-dessus. Il déplace aussi plusieurs passages, qu'il intègre à certaines démonstrations pour les renforcer ou les préciser. Les accroissements vraiment substantiels se réduisent à deux : à la suite de sa quadrature de la cycloïde, le Père Tacquet ajoute une démonstration à l'ancienne qu'on lui avait reproché d'omettre dans la Dissertatio de 1650 : d'autre part tous les derniers théorèmes relatifs à la manière dont s'opposent et se favorisent les mouvements qui composent Je mouvement cycloïdal sont la dilatation et l'explication d'une version originale beaucoup plus elliptique.

Un bref résumé de la Dissertatio de 1651 est ici nécessaire. Après l'avertissement Ad Lectorem, le Père Tacquet propose dix définitions, dont les principales sont celles de la volutio et de la rasio et la distinction des plans matériels commun, matériel parfait et géométrique. Suivent deux axiomes. Le Théorème I propose le problème fondamental : le paradoxe de la roue exposé par Aristote dans ses Problèmes mécaniques. Il consiste, comme on sait, en ce que deux cercles concentriques de rayons inégaux, dont l'un entrâne l'autre en roulant sur une droite, parcourent le même chemin sur cette droite. Un Scholie en dégage une série de six paradoxes secondaires, selon le Père Tacquet inconnus d'Aristote, de Galilée et de tous les géomètres. Ce premier mouvement s'achève sur une brève explication de la genèse des réflexions de l'auteur.

Dans les Théorèmes II à XVIII, le Jésuite envisage les différents rapports qui existent entre la circonférence du cercle roulant et la ligne qu'il accomplit sur le plan en une révolution. Les Théorèmes II à VII étudient le cas du plan géométrique abstrait. À partir du Théorème VIII en revanche on envisage les plans matériels, d'une part pour le roulement du cône (Théorèmes X à XIII), d'autre part pour la sphère, dans l'hypothèse d'une surface sphérique et d'un plan matériels parfaits (Théorèmes XIV à XV III). Les résultats sont assez différents du cas où le plan est géométrique. 
17 Le Théorème XIX ouvre une section consacrée à la cycloïde, dont le Père Tacquet donne une quadrature générale (Théorème XX), avec, dans le Scholie suivant, la démonstration apagogique complémentaire. Le Théorème XXI aborde un cas particulier de la construction d'une hypocycloïde (cas de $\mathrm{R}=2 \mathrm{r}$ ), sans élargir au cas général.

Les Théorèmes XXII à XXIX envisagent le mouvement du point qui trace la cycloïde, et, à partir d'une définition de l'opposition des mouvements, tentent de fixer les rapports des deux mouvements qui composent le mouvement du point traçant.

Dans cette abondante matière, le $\mathrm{P}$. Tacquet distingue, non sans prudence, les propositions qu'il estime rigoureusement démontrées de celles qu'il croit seulement probables (note suivant le Théorème XIII).

\section{Un géomètre casuiste}

La Dissertatio appartient au même genre du traité physico-mathématique que les Discorsi de Galilée qu'elle réfute, ou les Cogitata du P. Mersenne, pour ne citer que ces deux exemples célèbres. Le point de vue est assez proche de la méthode des mouvements de Roberval, par la conception générale du parallélogramme des mouvements comme par la distinction, dans le roulement du cercle, de deux mouvements composants de translation et de rotation. Cette perspective conduit l'auteur à s'intéresser plutôt aux paradoxes qui découlent des rapports entre les vitesses de ces mouvements et à la comparaison des lignes parcourues par le point qui se déplace sur la circonférence d'une part, et le centre d'autre part. Le point de vue de Pascal sera plus strictement mathématique, puisque le modèle de la balance ne lui sert qu'à introduire les sommes triangulaires dans un problème géométrique. Mais l'intérêt de la Dissertatio pour nous réside surtout dans la manière dont le Père Tacquet exploite cette perspective mixte. Dans son article sur l'Histoire de la Roulette, le Père Bosmans réduit la solution du paradoxe de la roue d'Aristote à bien distinguer et bien définir les idées de roulement et de glissement : on dit qu'une « circonférence animée d'un mouvement uniforme qui se déplace en touchant une droite roule sur cette droite quand sa longueur est égale à celle du segment qu'elle a parcouru après un tour entier ", et que « dans tous les autres cas elle glisse ; dans la roue d'Aristote, l'un des cercles roule, l'autre glisse ${ }^{14} »$. Mais justement le Père Tacquet n'envisage pas les choses ainsi. Il distingue bien la volutio du cercle, que l'on peut traduire par " roulement », et la rasio, qu'on peut être tenté de rendre par " glissement »; mais sous sa plume, ces termes ont un tout autre sens : la volutio consiste bien en un mouvement ininterrompu du cercle sur un plan, mais elle ne suppose pas que la circonférence du cercle est égale à la droite qu'elle parcourt sur le plan; autrement dit la volutio au sens du Père Tacquet enferme tout à la fois roulement et glissement. D'autre part la rasio suppose dans son esprit certaines discontinuités dans le mouvement de translation ou dans le mouvement de rotation du cercle. On pourra se convaincre à la lecture que cette distinction est loin d'être absurde, et qu'elle répond à des préoccupations voisines de celles qui inspirent la critique des indivisibles de Cavalieri. Mais c'est grâce à ces définitions de la volutio et de la rasio, fondement de tout l'édifice, que le Père Tacquet parvient à tirer du paradoxe initial une demi-douzaine d'autres mystères, dont il détaille les merveilles avec un visible plaisir, qui rappelle irrésistiblement celui du Jésuite des Provinciales, lorsqu'il exhibe les unes après les autres les mirifiques décisions des casuistes. D'autres paradoxes surgissent ensuite dans les Théorèmes IX et XI, dans le Théorème XV où i1 est montré qu'il est aussi naturel pour ne sphère de se déplacer sur un 
plan en roulant que sans rouler (Corollarium); même lorsqu'il approche le problème par lui-même intéressant de la génération de l'hypocycloïde (Théorème XXI), le Père Tacquet s'attache seulement au cas particulier paradoxal du mouvement de deux cercles qui engendrent une droite. La même inspiration baroque parait dans leur résolution, qui repose sur la distinction des plans matériel commun, matériel parfait et géométrique abstrait. Cette division n'a rien d'aberrant : elle répond dans les sciences à la nécessité de faire abstraction de certains aspects des phénomènes pour mieux les analyser ; c'est ainsi que l'on distingue géométrie, mécanique rationnelle et mécanique appliquée. Mais là aussi, on devine chez le Père Tacquet le goût du surprenant, de l'extraordinaire, une sorte de propension à la casuistique géométrique. Car il ne se sert guère des résultats établis dans l'ordre de la géométrie pure pour les appliquer au réel en spécifiant certains aspects par des données concrètes nouvelles: ce qui l'intéresse au contraire, c'est de montrer que, selon qu'on se situe dans l'abstraction géométrique, dans une réalité concrète supposée parfaite ou dans la réalité commune, les phénomènes du roulement varient parfois du pour au contre, un peu à la manière des casuistes qui se plaisent à prouver qu'une action est permise ou interdite selon qu'on envisage la spéculation ou la pratique. Cet esprit de casuistique éclate dans le Théorème $\mathrm{XV}$, signalé plus haut sur le mouvement d'une sphère sur un plan matériel parfait. Il y a de l'Escobar chez le Père Tacquet, et c'est à ce titre, beaucoup plus que pour ses inventions que la Dissertatio mérite qu'on la relise. Les Cylindricorum et Annulariurm Libri ou encore le Veterum Geometria promota du Père Lalouvère témoignent de l'attachement des mathématiciens jésuites pour la lettre des méthodes de l'Antiquité en même temps que de leur volonté de renouvellement ; mais la Dissertatio reflète un autre trait de l'esprit jésuite, non moins intéressant, parce qu'il est bien connu en dehors des mathématiques.

\section{BIBLIOGRAPHIE}

\section{Fuvres du Père Tacquet}

Dissertatio physico-mathematica de motu circuli et sphaerae. Quam praeside R.P. Andrea Tacquet, Societatis lesu Matheseos Professore defendit, explicuit ac demonstravit, Illustrissimus D. Philippus Eugenius comes de Hornes et d'Herlies, Lovanii 31 jan. Anno 1650, in Collegio Societatis Iesu. Lovanii, Typis Corn. Coenestenii, Anno 1650, in- $4^{\circ}$. BN : V6389 (2).

Cylindricorum et Annularium Libri IV ; item de circulorum Volutione per planum, Dissertatio physicomathematica. Antverpiae apud Jacobum Meursium, MDCLI, in-4º 288 p. BNV 6516 (l).

Ce dernier ouvrage comporte un livre supplémentaire dans l'édition de 1659. Les deux textes figurent dans les Opera mathematica de 1669 (1 vol. in-folio). 


\section{2. Études sur le Père Tacquet et la Dissertatio}

BOSMANS H., « Le Jésuite mathématicien anversois André Tacquet (1612-1660) », Gulden passer 3, 1925, p. 63-87.

BOSMANS H., « La notion des indivisibles chez Blaise Pascal », Archivio di Storia delle Scienza 4, 1923, p. 369-379.

BOSMANS H., «Sur l'œuvre mathématique de Blaise Pascal », Revue des Questions scientifiques, janv.-avril 1924, vol. 45, p. 130-161 et p. 424-451.

BOSMANS H., « Les premières pages de l'Histoire de la Roulette », Archives de Philosophie, I cahier 3, p. $92-112$

BOYER Carl B., The History of the Calculus and its conceptual Development, Dover Publications, NewYork, 1959, 346 p. (voir p. 139 sq.).

BUTZER P.L. et SCHAFFRATH, « Mathematics in Belgium from the Time of Charlemagne to the seventeenth Century », Actes du Colloque international « René-François de Sluse », Liège, 1986, 270 p. (voir p. 119 sq.).

MAUPIN G., "Quadrature de la Cycloïde d'après le Père Tacquet », Revue de Mathématiques Spéciales, III, 1894, p. 81-82.

GARDIES J.-L., Pascal entre Eudoxe et Cantor, Vrin, Paris, 1984, 144 p. (voir p. 49 sq.).

HARA, K., L'CEuvre mathématique de Pascal, Memoirs of the Faculty of Letters Osaka University, vol. XX I, Mars 1981, 240 p.

PASCAL B., Euvres complètes IV, CEuvres diverses (1657-1662), éd. Jean Mesnard, Desclée de Brouwer, Paris, 1992, 1708 p. (voir p. 399).

\section{Indications bibliographiques sur la roue d'Aristote}

ARISTOTE, Problèmes mécaniques, 24, 855 a.

GALILÉE, Discours concernant deux Sciences nouvelles, éd. Clavelin, Colin, Paris, 1970.

MERSENNE M., Les Mechaniques de Galilée, éd. Costabel et Lerner, Vrin, Paris, 1973.

AUGER Léon, Un savant méconnu : Gilles Personne de Roberval (1602-1675), Blanchard, Paris, 1962.

COSTABEL P., « La Roue d'Aristote et les critiques françaises à l'Argument de Galilée », Démarches originales de Descartes savant, Vrin, Paris, 1982.

Remarque : Sauf exception indiquée en note, les corrections apportées dans les errata ont été intégrées à la traduction.

\section{ANNEXES}

\section{Cylindricorum et Annularium Libri IV, Lib. II, Pars I}

Proposition VI 
Soit une sphère de diamètre $\mathrm{BC}$, de grand cercle $\mathrm{BICQ}$ et de centre $\mathrm{A}$.

On peut inscrire et circonscrire au demi-cercle BIC des moitiés de polygones réguliers, qui ont un côté parallèle au diamètre, comme GI et LM ; si on les fait tourner autour du diamètre $\mathrm{BC}$, ils inscrivent et circonscrivent à la sphère donnée des solides dont les surfaces diffèrent de la surface sphérique d'une quantité aussi petite que l'on veut.

Au polygone régulier OXGIZP, inscrit au demi-cercle BIC, on peut inscrire un autre cercle OFKP, qui touche tous les côtés du polygone ; et par conséquent les surfaces engendrées par le périmètre du polygone seront aussi circonscrites à la surface sphérique que produit le cercle OFKP, et néanmoins inscrites à la surface sphérique donnée BIZQ. Donc par ce que démontre Pappus, Livre V, Proposition 24, les surfaces produites par le périmètre XGIZ sont égales au cercle dont le rayon est le diamètre $\mathrm{OP}^{1}$. De la même façon, puisque par hypothèse les surfaces que le périmètre HLMN engendre sont circonscrites à la sphère donnée, ils seront aussi égaux au cercle dont le rayon est le diamètre $\mathrm{BC}$, par la même proposition de Pappus. Donc les surfaces engendrées par le périmètre HLMN sont aux surfaces engendrées par le périmètre XGIZ dans le même rapport que le cercle de rayon $\mathrm{BC}$ au cercle de rayon $\mathrm{OP}$, c'est-à-dire comme le carré de $\mathrm{BC}$ au carré de $\mathrm{OP}^{2}$. Mais les cercles engendrés par les rayons $\mathrm{HB}$ et NC sont aussi aux cercles engendrés par les rayons XO et ZP dans le rapport du carré de $\mathrm{BC}$ au carré de $\mathrm{OP}$ (en effet $\mathrm{HB}$ et NC sont à XO et ZP dans le rapport du périmètre $B H L M N C$ au périmètre OXGIZP ; c'est-à-dire, puisque les polygones sont semblables, comme $\mathrm{BC}$ à $\mathrm{OP}$ ); donc les surfaces engendrées par le périmètre entier BHLMNC, qui sont circonscrites à la sphère donnée BIZQ, sont à celles qu'engendre tout le périmètre OXGIZP qui sont inscrites à la sphère donnée, dans le rapport du carré de $\mathrm{BC}$ au carré de $\mathrm{OP}$, c'est-à-dire comme le polygone circonscrit au polygone inscrit ${ }^{3}$. Mais ces polygones peuvent différer du cercle BICQ d'une quantité aussi petite que l'on veut, comme le montre la Proposition 6 du premier livre d'Archimède De la Sphère et du Cylindre ${ }^{4}$. Donc les surfaces qui sont circonscrites et inscrites à la surface sphérique donnée BICQ peuvent aussi finir par différer de la surface sphérique d'une quantité aussi petite que l'on veut. Ce qu'il fallait démontrer.

\section{NOTES}

1. Euvres complètes, éd. J. Mesnard, II, p. 1271.

2. Bosman, « La notion des "indivisibles" chez Blaise Pascal », p. 374.

3. O.C, éd. Mesnard, IV, p. 438.

4. Voir Bosmans, op. cit., p. 377.

5. Voir Bosmans, «Sur l'œuvre mathématique de Blaise Pascal», I, p.131, pour l'édition de référence.

6. O.C., III, p. 368 sq.

7. O.C., II, p. 1270. Voir D. Descotes, «Les aspects littéraires et rhétoriques des Avertissements dans les Lettres de A. Dettonville de Pascal », dans La marge, Actes du colloque de Clermont-Ferrand, Clermont-Ferrand 1988, p. 109 sq.

8. O.C., IV, p. 438.

9. Voir ibid., Propositio XIV, p. 96. La Propositio VI se trouve p. 82-83.

10. Voir Ms. Fonds Français 9119, f 351 sq. Voir les remarques de J. Mesnard dans O.C. IV, p. 173.

11. Voir O.C., IV, p. 399.

12. Voir ces lettres dans O.C., IV, éd. J. Mesnard. 
13. Ces Elementa euclidea Geometriae planae ac solidae, Anvers, datent de 1654. Ils fournissent la liste complète des titres du dédicataire.

14. Bosmans, «Pascal et les premières pages de l'Histoire de la Roulette », p. 94. Voir les pages 96-97 consacrées au Père Tacquet

\section{NOTES DE FIN}

1. Voir Pappi Alexandrini Mathematicae Collectionesa Federico Command ino Urbinate in latinum conversae et Conunentariis illustratae, Pisauri, apud Hieronymum Concordiam, MDLXXXVIII, Theorema XXIII, Propositio XXIIII, f॰ 86 sq.

2. Le raisonnement est présenté ici sous une forme elliptique qui demande une brève explication. Toute la démonstration repose sur le fait, visible sur la figure, que si l'on ajoute à la surface engendrée par la ligne HLMN dans sa rotation autour du diamètre $B Q$, deux cercles de rayons égaux $\mathrm{HB}$ et $\mathrm{NQ}$, on engendre la surface produite par BHLMNQ tournée autour de BQ (et de même pour XGIZ et les cercles de rayons $\mathrm{OX}$ et $\mathrm{PZ}$ ). On vérifie facilement l'exactitude de la proposition en posant, comme le Père Tacquet, les deux proportions :

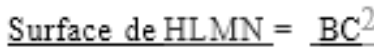

Surface de XGIZ OP ${ }^{2}$

et d'autre part :

$$
\frac{\text { Cercle de rayon } \mathrm{HB}}{\text { Cercle de rayon } \mathrm{XO}_{2}}=\frac{\pi \mathrm{HB}^{2}}{\pi \mathrm{XO}^{2}}=\underline{\mathrm{HB}^{2}}=\underline{\mathrm{BC}^{2}}
$$

Ces deux proportions peuvent être converties comme suit, puis additionnées :

$$
\begin{aligned}
& \text { Surf. HLMN. OP }{ }^{2}=\text { Surf. XGIZ. BC } \\
& +2 \mathrm{rC} \mathrm{HB}^{2} \cdot \mathrm{OP}^{2}=2 \pi \mathrm{X} 0^{2} \cdot \mathrm{BC}^{2} \\
& =\text { Surf. HLMN. OP } 2+2 \pi \mathrm{HB}^{2} \cdot \mathrm{OP}^{2}=\text { Surf. XGIZ. BC }{ }^{2}+2 \pi \mathrm{X}^{2} \cdot \mathrm{BC}^{2}
\end{aligned}
$$

Soit :

$\mathrm{OP}^{2}$. (Surface $\left.\mathrm{HLMN}+2 \pi \mathrm{HB}^{2}\right)=\mathrm{BC}^{2}$. (Surface XGIZ $\left.+2 \pi \mathrm{XO}^{2}\right)$

Et comme, si l'on ajoute à la surface engendrée par HLMN les deux cercles égaux de rayon HB et $\mathrm{NQ}$, on engendre la surface formée par BHLMNQ (et de même pour la surface engendrée par XGIZ et les cercles de rayon XO, qui forment la surface totale produite par la rotation de OXGIZP), on aura :

$\mathrm{OP}^{2}$. Surface BHLMNC $=\mathrm{BC}^{2}$. Surface OXGIZP

Et, comme le dit le Père Tacquet :

$$
\frac{\text { Surface BHLMNC }}{\text { Surface OXGIZP }}=\frac{\mathrm{BC}^{2}}{\mathrm{OP}^{2}}
$$

Mais ce raisonnement ne suit évidemment pas celui du Père Tacquet, qui consiste à tirer des deux proportions suivantes : 


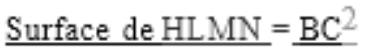 \\ Surface de XGIZ $\mathrm{OP}^{2}$}

Et

Cercle de rayon $\mathrm{HB}=\underline{\mathrm{BC}^{2}}$

Cercle de rayon $\mathrm{XO} \quad \overline{\mathrm{OP}^{2}}$

L'égalité suivante :

\section{$\underline{\text { Surface née de BHLMNC }}=\underline{\mathrm{BC}}^{2}$}

Surface née de OXGIZP $\mathrm{OP}^{2}$

Mais la conclusion paraît impossible, car il n'est pas vrai, à première vue, que :

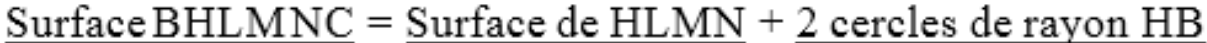 \\ Surface OXGIZP Surface de XGIZ 2 cercles de rayon XO}

Le Père Tacquet a sans doute en tête le raisonnement suivant :

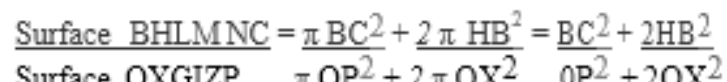

Ce dernier rapport ne diffère de $\mathrm{BC} 2$ / OP2 qu'en apparence, car on voit, sur les triangles semblables $\mathrm{AOF}$ et $\mathrm{ABF}$, que :

$$
\frac{\mathrm{BC}}{\mathrm{OP}}=\frac{2 \mathrm{BA}}{2 \mathrm{OA}}=\frac{\mathrm{BA}}{\mathrm{OA}}=\frac{\mathrm{BH}}{\mathrm{OX}}
$$

Et par suite :

$$
\frac{\mathrm{BC}^{2}}{\mathrm{OP}^{2}}=\frac{2 \mathrm{HB}^{2}}{2 \mathrm{OX}^{2}}
$$

Mais « deux grandeurs demeurent en même raison, quoiqu'on ajoute à l'une et à l'autre, pourvu que ce qu'on ajoute à la première soit à ce qu'on ajoute à la seconde, comme la première l'est à la seconde » (LAMY, Éléments des Mathématiques, III, sect. 3, Prop. III, p. 182). Par suite, si, comme on vient de le montrer :

On a aussi :

$$
\underline{\mathrm{BC}^{2}}=\frac{\mathrm{BC}^{2}+2 \mathrm{HB}^{2}}{\mathrm{OP}^{2}} \mathrm{OP}^{2}+2 \mathrm{OX}^{2}
$$

La démonstration est donc exacte malgré son caractère elliptique.

3. Le rapport des deux polygones est identique au rapport des triangles OAF et BAH. Donc:

$$
\frac{\text { Polygone circonscrit }}{\text { Polygone inscrit }}=\frac{1 / 2 \mathrm{AB} \cdot \mathrm{BH}}{1 / 2 \mathrm{AO} \cdot \mathrm{OX}}=\frac{\mathrm{AB} \cdot \mathrm{BH}}{\mathrm{AO} \cdot \mathrm{OX}}
$$

Mais à cause des triangles semblables, on a :

$$
\frac{\mathrm{AB}}{\mathrm{AO}}=\frac{\mathrm{BH}}{\mathrm{OX}} \text { soit } \frac{2 \mathrm{AB}}{2 \mathrm{AO}}=\frac{\mathrm{BC}}{\mathrm{OP}}
$$


Donc :

\section{$\underline{\text { Polygone circonscrit }}=\underline{\mathrm{AB}} \cdot \mathrm{BH}=\underline{\mathrm{HB}} \cdot \mathrm{HB}=\underline{\mathrm{HB}^{2}}$ \\ Polygone inscrit AO.OX OX. OX $\mathrm{OX}^{2}$}

4. Voir le commentaire de Heath, A History of greek Mathematics, II, p. 35-36, sur cette proposition.

INDEX

Mots-clés : Pascal, Tacquet (Père), Lettres de A. Dettonville, mathématiques, geométrie

Keywords : Pascal, Tacquet (Father), Lettres de A. Dettonville, mathematics, geometry

AUTEUR

DOMINIQUE DESCOTES

CERHAC, Université Blaise Pascal 